\title{
Washing Durability of Cotton Coated with a Fluorinated Resin: An AFM, XPS, and Low Frequency Mechanical Spectroscopy Study
}

\author{
D. Juhué, A. C. Gayon, and J. M. Corpart \\ Atofina, Centre d'Application de Levallois, 92300 Levallois.Perret, France \\ C. QUET \\ Alofina. Groupement de Recherche de Lacq, B.P. 34, 64I70 Artix. France \\ P. DELICHÈRE \\ IRC, UPR CNRS, 69626 Villeurbanne, France \\ N. Charret, L. David, J. Y. Cavaillé, and P. Perriat ${ }^{1}$ \\ GEMPPM. UMR 5510 CNRS - INSA de Lyon. 69621 Villeurbanne. France
}

\begin{abstract}
The evolution, during laundering, of mechanical, chemical. and morphological properties of cellulose fibers covered with a fluorocarbon resin is studied using a combination of low frequency mechanical spectroscopy (LFMS), x-ray photoelectron spectroscopy (XPS), and atomic force microscopy (AFM). From this study of the roughness and surface composition of the film covering cellulose fibers, new explanations are proposed for the decrease in water and oil repellency with washing, and its partial recovery with subsequent heat treatment such as ironing. The observed phenomena involve de-wetting and rewetting of the fluorinated polymer on the cellulose surfaces. inducing a modification of adhesion mechanisms between the fluorocarbon polymer and the cellulose fibers and the formation and/or destruction of fluorinated polymer bridges (matrix or ligand effect) between the fibers.
\end{abstract}

The performance and durability of modern fabric tinishes require improved laundry permanence of water and oil repellent finishes. This can be obtained by coating natural textiles with acrylic copolymers containing fluorine; indeed, these fluorocarbons not only confer appropriate hydrophobic and oleophobic properties [6.11], but also suitable affinities with cellulose for providing highly adhesive, uniforn. permanent coatings [25]. As a consequence, fluorine-treated clothes can be subjected to more than thirty laundry cycles [6] without significant evolution of waterproof or shrinkage resistance. Some researchers have documented. however, that water repellency decreases with washing and partially recovers with ironing $[1,14,27,28]$. Such reversible effects are generally explained by the rotation of fluoroalkyl groups into the polymer substrate to avoid the hydrophilic conditions during washing [28]. In this paper, we propose an alternative explanation: Based on massive de-wetting (respectively, wetting) of fluorinated resin at the fiber surface during washing (respectively, ironing), it involves a morphology change of the polymer/fiber interface. We assess this new interpretation using a combination of different techniques that permit a correlative evolutionary study of microstructural, chemical, and mechanical properties during laundering. In particular, we determine the mechanical properties of fluorinated resin-treated cotton for the first time using the technique of forced torsion low frequency mechanical spectroscopy (LFMS).

Two original facts directly related to the wetting properties of fluorocarbons on cellulose can be drawn from these new experiments. First, the measured stiffness of the textile gives some direct indication of the adhesion between the fibers and the fluorinated film because a strong adhesive coating increases the stiffness of each . liber and thus the stiffness of the fabric. This point is true even if deriving the mechanical properties of textiles 
from those of its fibers is a rather complicated problem [26]. Second, stiffness also directly depends, in correlation with de-wetting. on the eventual formation of some polymer bridges between the cellulose fibers. Moreover. since textile stiffness has no reason to depend on the rotation of the fluoroalkyl groups, the mechanical spectroscopic experiments may be used to discriminate the respective contributions of the fluorocarbon rotation and the wetting/de-wetting mechanisms to modifications of water and oil repellency during laundering. In addition to this technique, we use $\mathrm{x}$-ray photoelectron spectroscopy (XPS) to confirm our proposed interpretation by assessing the surface chemical properties, and atomic force microscopy (AFM) to obtain information on the roughness of the polymer film deposited on the fibers. In panicular, we compare XPS results with the features of a simple model proposed to calculate changes in the textile surface composition due to de- and re-wetting mechanisms.

\section{Experimental}

\section{MATERIALS}

Cotton fibers used as the substrate for the polymer coating (specific area of $270 \mathrm{~m}^{2} / \mathrm{g}$ ) came from Crouvezier. France. They mainly had a crystalline structure of cellulose I with high crystalline content and a small residue of cellulose II.

The cationic latex dispersion (latex A) had polymer particles about $100 \mathrm{~nm}$ diameter of a fluoroalkyl ethyl acrylate $(50-x$ mol. \%) co-behenyl acrylate (50 - y mol. \%) co- $\mathrm{N}$-methylol acrylamide $(x+y<20$ mol. \%). This blend composition was a compromise [4] for oil and water repellency (by means of the density of $\mathrm{CF}_{3}$ terminal groups) and further bonding on cellulose fibers (by means of the density of $\mathrm{OH}$ terminal groups). Fluorine-containing acrylic copolymers were then synthesized by classical emulsion polymerization using acetone as the cosolvent. The copolymer so obtained had a double period comb-structure [17\}, altemating layers of sidefluorinated chains containing an average number of $8 \mathrm{~F}$ atoms and side-hydrocarbonated chains with 18-22 C atoms. Another copolymer (latex B) with a higher glass Iransition temperature was synthesized using vinyl chloride $(30 \mathrm{~mol} \%)$ as a co-monomer to replace part of the behenyl acrylate. For latices $\mathrm{A}$ and $\mathrm{B}$. the solid content was fixed at $20 \%$.

Cotton fabrics were padded with 6 and 15 water $\%$ of fluorinated acrylic dispersions and squeezed to about 90\% wet pickup. Treated fabrics were then thermally dried and cured for 3 minutes at $150^{\circ} \mathrm{C}$.

\section{INSTRL'MENTATION}

Torsion mechanical spcctroscop:: Low frequency mechanical analysis (LFMA) [8] was measured on a homemade high resolution apparatus $\{9.29\}$ specifically designed to study samples of low rigidity: it consists of a forced oscillation pendulum working in the temperature range of $100-500 \mathrm{~K}$ and frequency range of $5 \times 10^{-5} 10$ $5 \mathrm{~Hz}$. Since the maximum strain was less than $10^{-4}$, the viscoelastic behavior of the sample was linear (i.e.. independent of the applied stress for all temperature ranges used for experiments). The storage $\left(K^{\prime}\right)$ and the loss $\left(K^{\prime \prime}\right)$ of complex shear stiffness $\left(K^{*}\right)$ and so internal friction $\tan \Phi=K^{\prime \prime} K^{\prime}$ were measured as a function of temperature at 0.3 and $1 \mathrm{~Hz}$. These values were a good compromise betwoen the time needed for experiments and elimination of resonance phenomena [13]. In these conditions. resolution was better than $\tan \Phi=5 \times 10^{-4}$, and apparatus contribution to rigidity could be neglected thanks to careful design. Sample dimensions were about $5 \mathrm{~mm}$ long. $2 \mathrm{~mm}$ wide. and $200 \mu \mathrm{m}$ thick.

The apparent complex rigidity $\boldsymbol{K}^{*}$ does not provide direct comparative information about the viscoelastic properties of the fabrics because of slight differences in the geometry of the different samples. Considering that at high temperatures. the influence of the fluorinated resin on the measured stiffness is negligible. stiffness values were then normalized to the same value at the highest temperature investigated. $200^{\circ} \mathrm{C}$. This correction is consistent with that expected from the evaluation of the sample dimensions and is always less than $10 \%$.

$X P S$ analysis at the Atofina Research Center involved an SSI-SX 100 semi-hemispheric analyzer with $220 \mathrm{~W} \mathrm{AI}$ $\mathrm{K}_{\alpha}$ radiation $(1486.6 \mathrm{eV}$ ). All spectra were confirmed at the Institut de Recherche sur la Catalyse with a VG Scientific ESCA LAB $200 \mathrm{R}$ using the same radiation at 240 $W$. In both cases. the samples were $1.5 \times 1 \mathrm{~cm}$ pieces of cotton voile fabric with a spot size on the sample of 400 $\times 1000 \mu \mathrm{m}^{2}$. Emerging photoelectrons were analyzed with the SSI-SX with detection at $35^{\circ}$ and normal to the sheet surface with the vG apparatus. Spectra relative to $\mathrm{C}_{1}, \mathrm{O}_{1}$. and $F_{1}$, photoelectron peaks were measured at binding energies of around 285.0 [16]. 583. and $688 \mathrm{eV}$. respectively. The resolution in energy for the carton element was $1.2 \mathrm{eV}$. After smoothing, backgrounds were removed by the $S$ hirley routine [24]. The total area of $C_{1}$ and $F_{1}$, peaks and satellites (areas $C$ and $F$ ) allowed us to calculate the atomic C/F-ratio according to the formula [5]

$$
\frac{C}{F}=\frac{k, \text { area } C}{k, \text { area } \mathrm{F}} .
$$

where $k_{\text {, and }} k_{f}$ are the $\mathrm{C}$ and $\mathrm{F}$ sensitivity factors. 
Sensitivity factors contain the photoemission cross sections as approximated from a Hartree-Slater atomic model [20]. With the ssI-sx 100 semi-hemispheric analyzer, these factors were conected by a factor proportional to $\left(E_{\mathrm{kin}}\right)^{0.2}$, which is the photoelectron kinetic energy, while with the ESCA LAB $200 \mathrm{R}$, they were corrected by a factor proportional to $\left(E_{\mathrm{kin}}\right)^{0.1}$. Attenuation from the free electron mean path [21] and from the transfer function of the spectrometer [22] were then accounted for. As a consequence of all these approximations, absolute uncertainties in XPS quantitative analysis are generally of the order of $\pm 10 \%$. However, experimental reproducibility is better than $\pm 3 \%$, so that when considering only comparisons between samples, the relevant unceltainties are less than $\pm 3 \%$.

AFM (atomic force microscopy): All samples were imaged with an Autoprobe CP apparatus from Park Scientific Instrument. Samples were scanned in the tapping mode by an ultralever (ULNC-AUNM) tip with a constant force of about $2.1 \mathrm{~N} \cdot \mathrm{m}^{-1}$ and a resonance frequency of $100 \mathrm{kHz}$.

Fluorine content meusurement: Fluorinated resintreated fabrics were burnt under pure oxygen in a Schocniger flask. Liberated fluorine was absorbed in a solution of sodium hydroxide $(0.1 \mathrm{~N})$; the solution was then titrated using a fluoride selective electrode.

Oil repellency: Oil resistance was measured according to the American Association of Textile Chemists and Colorists (AATCC) Test Method 118-1992. In this test, oily drops are deposited on a substrate, allowed to remain for 30 seconds, and removed by wicking or wiping with a paper tissue. The substrate is then observed for remaining signs of wetting. Eight different challenge liquids with different liquid tensions are numbered by a rating increasing from 1 in the case of vaseline, the easiest to repel, to 8 in the case of $n$-heptane, the most difficult to repel (Table 1). Oil repellency is reported as the maximum rating of the liquid that does not wet the substrate. For detailed comparisons, multiple drops of each liquid are tested. In general, an oil repellency rating of 5 or higher is desirable.

TA8LE I. AATCC Test Method 118-1992: definition of oil repellency rating.

\begin{tabular}{clc}
\hline $\begin{array}{c}\text { Oil repellency } \\
\text { rating }\end{array}$ & \multicolumn{1}{c}{ Test liquid } & $\gamma_{L}, \mathrm{mN} / \mathrm{m}$ \\
\hline 8 & n-heplane & 19.8 \\
7 & n-octane & 21.4 \\
6 & n-decane & 23.5 \\
5 & n-dodecane & 24.7 \\
4 & n-teradecane & 26.4 \\
3 & n-hexadecane & 27.3 \\
2 & 35/65 mix of n-hexadecane/vaseline & 29.6 \\
1 & Vascline & 31.5 \\
\hline
\end{tabular}

Water repellency: The water spray test follows AATCC Test Method 22-1989. During the test. the substrate is held taut within a $15 \mathrm{~cm}$ diameter ring at a $45^{\circ}$ angle, and $250 \mathrm{~mL}$ of water at a temperature of $23 \pm 1^{\circ} \mathrm{C}$ are dropped onto the substrate from a distance of 15.2 $\mathrm{cm}$. The substrate is then tapped lightly to remove excess water and is rated by determining the pattem of surface wetting. A higher number indicates better water repellency and, in general. a rating of $\mathbf{8 0}$ or higher is desirable.

\section{Results and Discussion}

\section{Physical Interpretation of the Evolution of Complex Rigidity With Temperature}

All relaxations. phase transitions. and chemical reactions provide some contribution to the temperature evolution of the complex rigidity $K^{*}$ of the fabrics: to be precise, they generally involve peaks in the mechanical losses curves at the temperature where they take place. For the sake of clarity, heating scans will be noted $K^{*}(T \uparrow)$ and cooling experiments $K^{*}(T \downarrow)$. Examples of $K^{\prime \prime}(T \uparrow)$ curves Figure $1 \mathrm{~b}$ for pure and coated cotton fabrics show that the mechanical loss curves present two more or less pronounced major peaks. one at around $-45^{\circ} \mathrm{C}$ and the other between 10 and $50^{\circ} \mathrm{C}$. We have elucidated the physical origin of these peaks with help of additional experiments.

\section{$\beta$ Relaxation of Amorphous Cellulose at $-45^{\circ} \mathrm{C}$}

The $\tan \Phi$ peak at $-45^{\circ} \mathrm{C}$. which is present for each cotton fabric, coated or not. may be associated with the $\beta$ relaxation of the amorphous cellulose [15]. This conclusion is supported by the fact that the observed relaxation temperature corresponds to that relative to the standard state of cellulose under normal atmospheric conditions: indeed, $T_{\theta}$ is repolted to vary in the range -40 to $-80^{\circ} \mathrm{C}$ when the $\mathrm{H}_{2} \mathrm{O}$ content varies from 0 to $6 \%$ [15]. This $\beta$ relaxation temperature shift with increasing $\mathrm{pH}_{2} \mathrm{O}$ is explained by the correlative increase of the mobility of the cellulose chains due to the plasticizing effect of water [19]. which prevents intermacromolecular $\mathrm{H}$-bonds. To reinforce the conclusion that the peak at around $-45^{\circ} \mathrm{C}$ may be attributed to cellulose $\beta$ relaxation. we studied two pure cottons with different water contents. We found a difference of about $10^{\circ} \mathrm{C}$ between the temperature of the $\beta$ relaxation for, on the one hand. the cotton containing more humidity [dotted line in Figure 2a relative to the measure of $K^{*}(T \downarrow)$ under nitrogen of a pure cotton initially at ambient conditions] and for, on the other hand, the driest cotton (bold curve Figure 2a relative to the measure of $K^{*}(T \uparrow)$ under vacuum of a pure cotton previously cooled under 


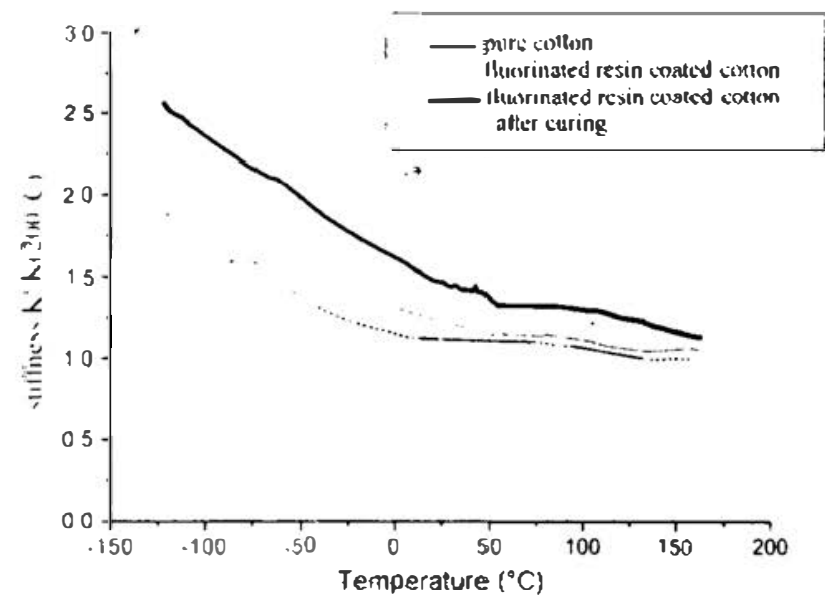

(a)

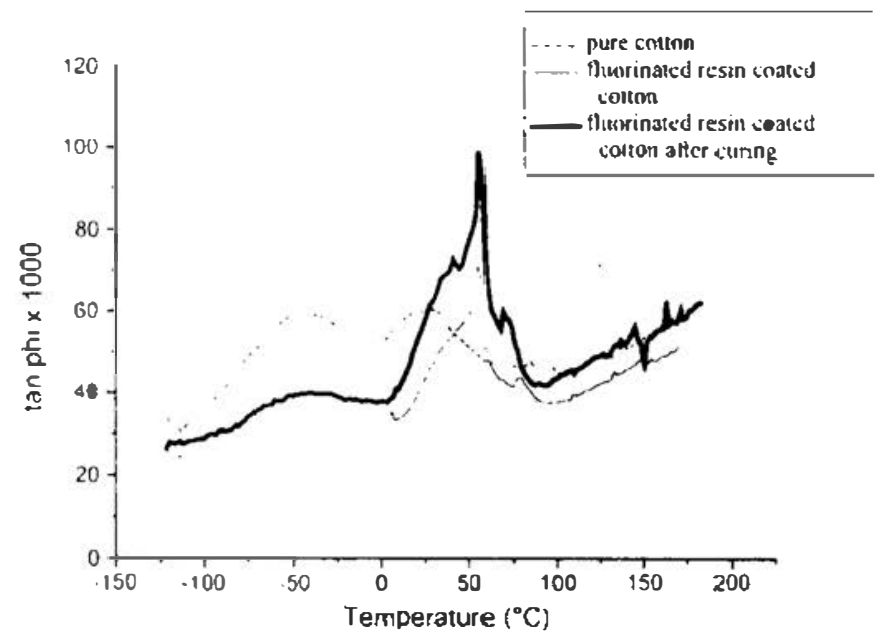

(b)

Fuil'RE 1. Normalized stiftness (a) and $\tan \phi$ (b) of pure and fluorinated resin-coated cotton fabrics measured by a corsion mechanical spectrometry experiment as a function of temperature during heating up to $160^{\circ} \mathrm{C}$ at $1^{\circ} \mathrm{C} / \mathrm{min}$ under air. The polymer-coated textiles are studied befure and atter thermocuring for 3 minutes at $150^{\circ} \mathrm{C}$.

nitrogen]. Such a difference between the $T_{\beta}$ values is. consistent with the difference in water content $(\approx 1 \%)$ expected for the two cellulose samples studied under vacuum and under nitrogen.

\section{Contributions to Peaks in the $10-50^{\circ} \mathrm{C}$ Temperature Range}

Due to hydrogen bonds. cellulose libers are water absorbent $[1,12,23]$. We thus attribute the second peak located between 10 and $40^{\circ} \mathrm{C}$ in the mechanical losses curves of pure cotton fabrics to water removal (Figure 2c). This result is confirmed by comparing the evolution of the complex rigidity of pure cotton fabrics during heating up to $100^{\circ} \mathrm{C}$ under vacuum and further cooling from $100^{\circ} \mathrm{C}$ to room temperature under vacuum: indeed. there is a complete disappearance of the peak after all the water in the cotton has evaporated. Attributing the sec-
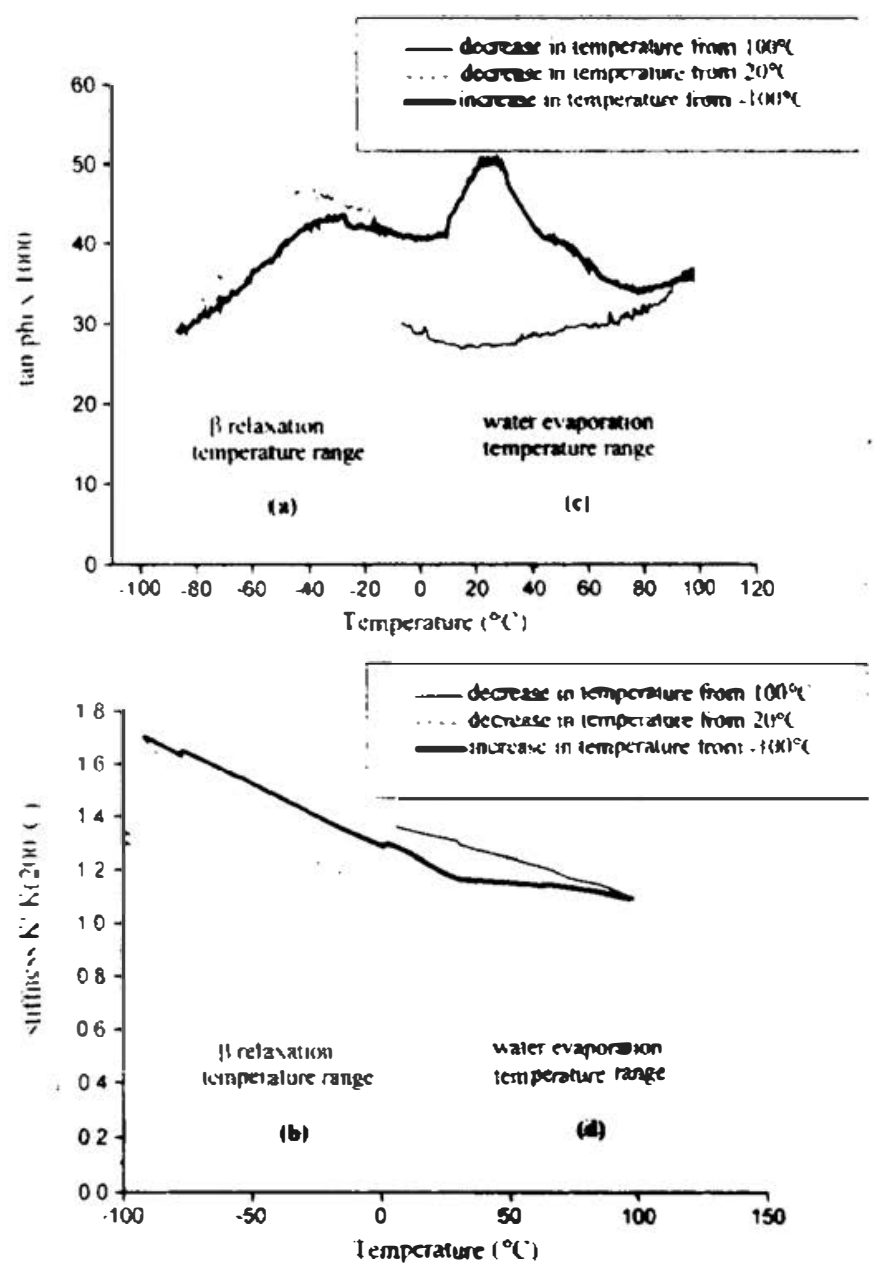

FIGL:RE 2. Effect of theinnal treatment (heating at $I^{\circ} \mathrm{C} / \mathrm{min}$ followed by cocoling at the same rate) upon the stiffness $(b$ and $d$ ) and $\tan \Phi$ (a and $c$ l of a pure cotton fabrics. In the $a$ and $b$ zones. $\beta$ retaxation of pure celfulose is observed for cooling under nitrogen and for heating under vacuum. In the $\mathrm{c}$ and $\mathrm{d}$ zones. one experiment corresponds to heating under vacuum up to $100^{\circ} \mathrm{C}$. Since the tan peak at about $30^{\circ} \mathrm{C}$ is not observed during cooling under vacuum in view c. this peak can be attributed to water evaporstion.

ond peak to water removal from cellulose is also consistent with the fact that the position of such a peak does not depend on frequency. as it would in the case of any relaxation phenomenon.

In the case of fluorinated resin-coated cotton. there is another shaip contribution near $50^{\circ} \mathrm{C}$ in the second peak of the tan $\Phi$ curves (Figure 1). This contribution coincides with the melting transition [7] of the crystallite pan of the fluorinated polymer characterized by a wide transition between a low mesomorphic - highly organizedphase and a high temperature one. Attributing the sharp part of the second peak to the melting transition of the fluorinated resin is also confirmed by calorimetric measurements and further considerations about the evolution of the melting peak amplizudes and positions with frequency. Indeed. for coated fabrics, the amplitude of the sharp part of the peak decreases with frequency $f$, while 
its temperature is not sensitive to $f$, which is effectively consistent with a phase transition melting process [18].

\section{EFfect of the Coating on Fabric Mechanical Properties}

The effect of coating on the complex rigidity $K^{*}$ of the fabrics can be evaluated by comparing the evolution of $K^{\prime}$ and $K^{\prime \prime}$ with temperature on three different samples: a pure cotton sample, a polymer-coated cotton sample. and a polymer-coated then cured cotton sample (Figure 1). The main result is that the apparent elastic and viscoelastic properties of the fabrics are significantly modified by the presence of the polymer coating: this is visible in Figure la with a higher normalized stiffness $K^{\prime}$ in the case of the coated textiles. It is also noticeable in the low temperature range. Figure Ib. where the mechanical losses $K^{n}$ of the cotton fabrics correlatively diminish. This last point is consistent with the absence of relaxation processes of the highly crystalline fluorinated resin at temperatures below the melting transition.

\section{Fiber-Resin Adhesion and Formation of Resin Bridges Berveen Fibers}

We propose two explanations for the increase of around $30 \%$ of the norinalized stiffness of fabrics after coating and curing (Figure 1). One is at the level of the cotton fiber, and the second is relative to the formation of resin bridges between the fibers. At the level of the fiber modeled by a cylinder with momentum $I=\frac{\pi R^{\prime}}{4}(R$ being the radius of the cylinder). an additional perfectly adhesive shell of $0.5 \mu \mathrm{m}$ on the surface increases the momentum of a fiber of $10 \mu \mathrm{m}$ by a factor of around $20 \%$. Great uncertainties remain. however, about the stiffness increase itself, since stiffness also contains the Young's modulus of the inner fiber and the outer coating. Assuming that the order of magnitude of the Young*s modulus of the resin is equivalent to that of poly(tetrafluoroethylene): $\approx 0.35 \mathrm{GPa}[2,4]$ and that of cellulose is about $20 \mathrm{GPa}$ [4]. the stiffness increase of each fiber due to the presence of the adhesive coating would be much less than $20 \%$. As a consequence, the correlative stiff. ness increase of the fabric itself would also be much less than $20 \%$. Since the experimental increase of stiffness is around $30 \%$, another contribution rather than that arising from the individual increase of each fiber with a coating has then to be considered. The second explanation for the increase of $K^{\prime}$ is related to some ligand effect (bridge formation) of the fluorinated resin [14] above the melting temperature, and we will discuss that in detail below.

\section{Comparison of $\mathrm{K}^{\prime}$ and $\mathrm{K}^{\prime \prime}$ of Coated Fabrics.}

\section{Cured or Nol}

Even if the stiffness values of polymer coated cotton with and without curing are higher than in the case of pure cotton (Figure la), the increased stiffness is more pronounced in the temperature range -150 to $50^{\circ} \mathrm{C}$ when the fluorinated resin-treated cotton has been cured at $150^{\circ} \mathrm{C}$. This is consistent with the fact that both the latex particle interdiffusion providing resin cohesion and the cotton/resin adhesion are probably concomitant with polymer melting $[10]$ at $50^{\circ} \mathrm{C}$. This temperature of $50^{\circ} \mathrm{C}$ then has to be overcome before the stiffness of the coated fabrics significantly increases. Just after bathing in the colloid and room temperature drying, about five or six layers of latex particles of about $100 \mathrm{~nm}$ are deposited on the cotton, but with poor mechanical cohesion and weak adhesion to the fibers. That explains why there is only a slight increase in the stiffness after washing and drying (Figure la). In fact, after heating during a short time at $150^{\circ} \mathrm{C}$. the crystalline part of the latex particles melts and the consecutive resin viscosity drop enables resin spreading and adhesion with cellulose.

The melting peaks between 50 and $70^{\circ} \mathrm{C}$ (Figure $\mathrm{lb}$ ) are also more evident when the coated textile has been cured at a high temperature: this has to be related again to the increased adhesion of the polymer film with cellulose and to the formation of a polymer matrix between fibers, both phenomena enhancing the transmission of the torque applied to the polymer coating. For higher temperatures, there is no more difference in the $\tan \Phi$. curves relative to pure or polymer-coated, cured or not, cotton fabrics. Indeed, above the crystallite melting temperature, the small rigidity of the fluorinated polymer implies that the mechanical torque is transmitted almost entirely by the cotton. so the polymer makes no significant contribution to dissipative phenomena.

\section{Confirming the Interpretation with AFM and MEB}

The evolution of fibers molphology with coating and curing is directly evident from AFM and MEB. As shown in the MEB micrograph of Figure 3a, the shape of the pure cotton fibers is flat with a width of $10 \mu \mathrm{m}$. The evolution of the fiber's morphology is followed in the AFM using a window of $4 \times 4 \mu \mathrm{m}$. thus allowing the roughness characterization of each individual fiber. In Figures $3 b$ and $4 c$, whereas the roughness of a pure cotton fiber is about $50 \mathrm{~nm}$. that of an uncured polymer-coated cotton fiber increases up to $\approx 300 \mathrm{~nm}$. The latter value is consistent with the average thickness of the fluorocarbon film of about $500 \mathrm{~nm}$. As shown in Figures 3c-d, the thickness heterogeneities are almost completely suppressed by curing, so that the roughness of the fiber after curing is approximately the same as for pure cotton. 


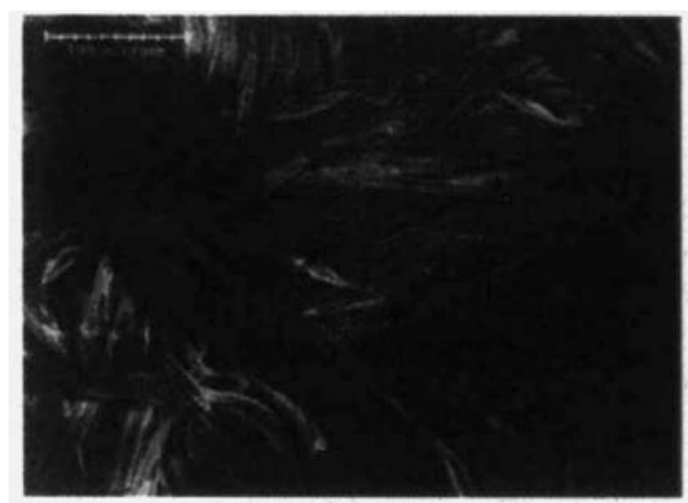

(a)

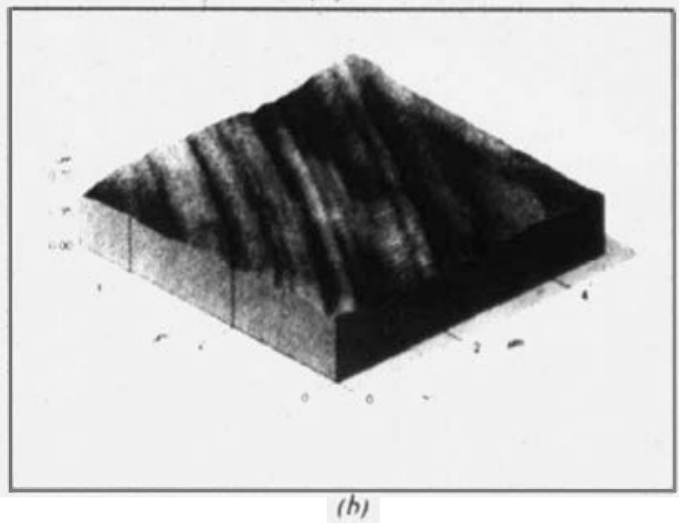

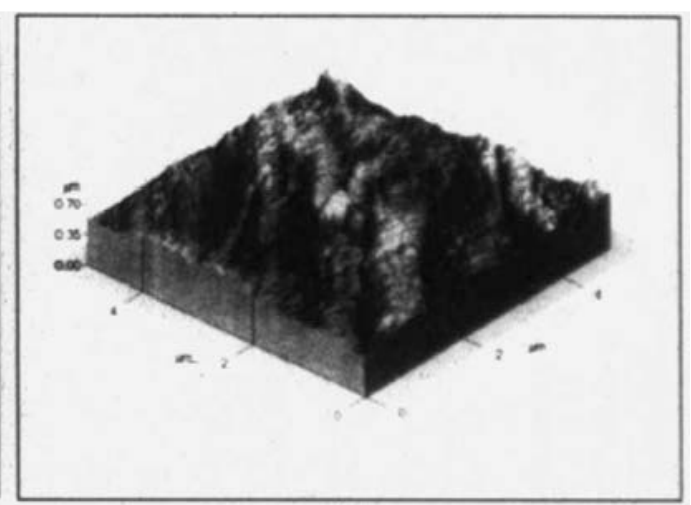

(c)

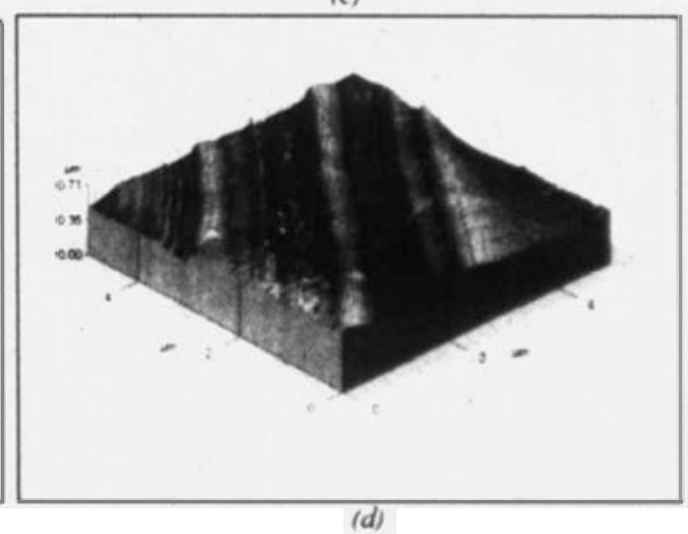

FIGLiRE 3. AFM surface investigations of pure colton (b), MEB and AFM surface investigations of fluorinated resin-coated cotton before cusing (a and c). and AFM surface investigations of florinated resin coated cotton atces curing (d). An increase in roughness is noticeable after polymer deposition on corton fibers. This increase is almost entirely removed by thermal treatment at $150^{\circ} \mathrm{C}$

\section{EFFECT OF WASHING/IRONING}

Stiffness and Mechanical Losses of Washed and Ironed Coased Texuiles

In order to evaluate the influence of washing and ironing on the mechanical properties of coated fabrics. we measured the complex modulus of washed and ironed coated textiles and compared it with that for the textile after curing. Compared with the coated and cured textile, a noticeable decrease in the stiffness of the washed textile is shown in Figure 4. In accordance with many results in the literature [28], some recovering of cotton stiffness occurs after ironing. This last result is perfectly true out of the -100 to $0^{\circ} \mathrm{C}$ temperature range, whereas between -100 and $0^{\circ} \mathrm{C}$, the stiffness values of both washed and ironed coated textiles are only equivalent. However, we have already proved that the $\boldsymbol{\beta}$-relaxation of cellulose between -100 and $0^{\circ} \mathrm{C}$ is very sensitive to $p \mathrm{H}_{2} \mathrm{O}$ : considering that the water partial pressure $\mathrm{pH}_{2} \mathrm{O}$ is not fully controlled during the experiments, we may expect some $K^{\prime}$ uncerainties related to $\mathrm{pH}_{2} \mathrm{O}$ fluctuations of about $5 \%$ in the -100 to $0^{\circ} \mathrm{C}$ temperature range. Some relevant comparisons of samples with modulus values differing from $5-20 \%$ (which is the case for the washed and ironed coated textiles considered here) should then be relevant only below $-100^{\circ} \mathrm{C}$ and above $0^{\circ} \mathrm{C}$ : this corresponds effectively to the temperature range in which the stiffness of the ironed coated textile is, as expected. higher than that of the washed one (Figure 4a). The mechanical loss amplitude relative to the peak at $50^{\circ} \mathrm{C}$ undergoes similar variations: tan decreases from $61 \times 10^{-3}$ to $45 \times 10^{-3}$ during washing and shows some further recovery up to $55 \times 10^{-3}$ afier ironing.

\section{De-wetling During Washing, Re-wetting During Ironing}

Variations in stiffness and tan $\Phi$ induced by washing and ironing can be explained by wetiability considerations. During washing, the evolution of a cellulose/ resin/ $\mathrm{H}_{2} \mathrm{O}$ system has to be considered, whereas it is a cellulose/resin/dry gas system during ironing. This corresponds to conditions of complete immersion of the textile in water during washing and a dry atmosphere during ironing. During washing, some resin de-wetting from cotton fibers can be justified by thermodynamic considerations: indeed. the resin $/ \mathrm{H}_{2} \mathrm{O}$ interface energy is 


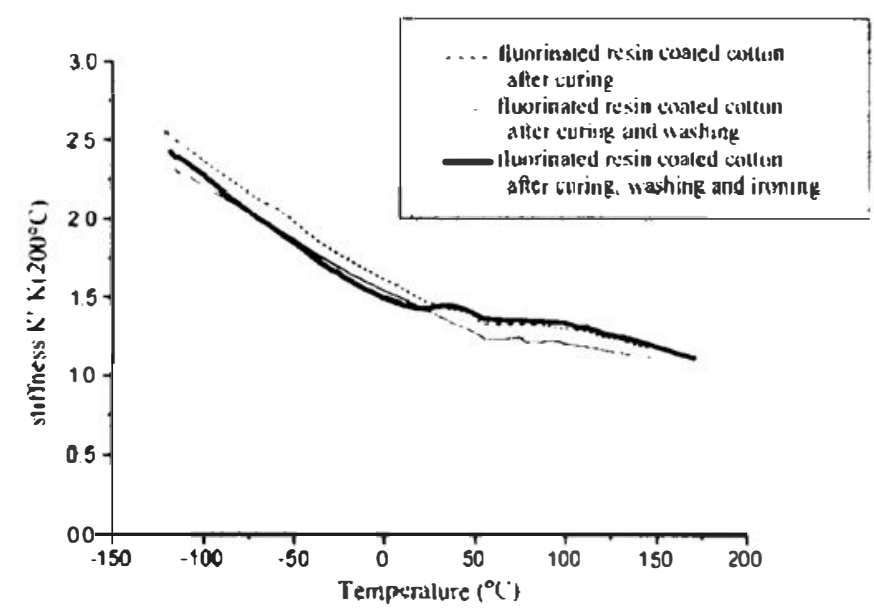

(a)

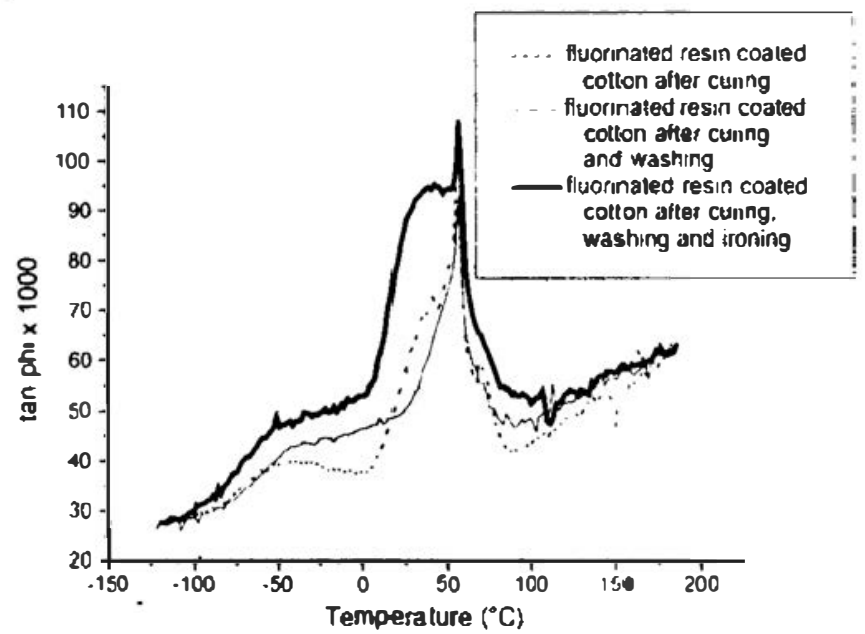

(b)

Figuke 4. Stiffness (a) and tan $\Phi$ (b) of a flumrinated resin-coated cotton measured by a torsion mechanical spectrometsy experiment ats a function of temperalure Juring heating up in $150^{\circ} \mathrm{C}$ al $1^{\circ} \mathrm{C} /$ min under air. The coated fabrics are studied after curing in $150^{\circ} \mathrm{C}$. after three washings at $40^{\circ} \mathrm{C}$. and after ironing at $150^{\circ} \mathrm{C}$.

high due to the hydrophobic properties of $\mathrm{CF}_{2}$ and $\mathrm{CF}_{3}$ entities, whereas the cellulose/ $\mathrm{H}_{2} \mathrm{O}$ interface energy is low due to the hydrophilic character of cellulose. In accordance with these surface energies, we then expect during washing the rupture of the uniforn resin coating on the surface of the fibers and the concomitant appearance of cellulose at the textile surface in contact with water. De-wetting, which involves a diminution of the cellulose/resin adhesion, is then consistent with the stiffness decrease with washing. It is also consistent with the decrease of the second peak amplitude in the $\tan \Phi$ curves. since a smaller contuibution of the melting transition of the resin to the losses is expected when the adhesion between cellulose and resin is poor. During ironing, there is a complete melting of the coating which. due to the low resin viscosity at high temperature. should evolve to the thermodynamically favored state. The resin/ dry gas interface has lower energy than the cellulose/dry gas one, which contains some polar atoms (oxygen). The more favored state of the cellulose/resin/dry gas system should then correspond to uniform spreading of the resin at the surface of the fibers. This increased adhesion between cellulose and resin is concomitant with the fornation of resin bridges between the fibers, both phenomena explaining the stiff ness and the $\tan \Phi$ increases observed after ironing.

\section{Relative Consributions of Fiber-Resin Adhesion and Resin Bridge Formation to Complex Kigidity}

De-wetting and re-wetting may appear as relatively small effects when they are evidenced by the previously described mechanical spectroscopic experiments. A possible explanation for such small differences in $K^{\prime}$ and tan $\Phi$ between textiles subjected to different treatments may originate from the experimental procedure we followed. Indeed, each sample is prepared "ex-situ" before being studied inside the pendulum. It is then possible that some of the resin bridges created between the cotton tibers during ironing may be broken by further manipulations until mechanical measurements. That would explain in particular why the stiffness of the textile has been improved by a factor of only a few percent after ironing. This point is justified by reproducing "in-situ" the conditions of ironing (Figure 5). In this in-situ ironing process. a polymer-coated cotton fabric. thermally cured and washed. is studied with LFMA during heating at $1{ }^{\circ} \mathrm{C} / \mathrm{min}$ up to the usual ironing temperature $\left(150^{\circ} \mathrm{C}\right)$ and then

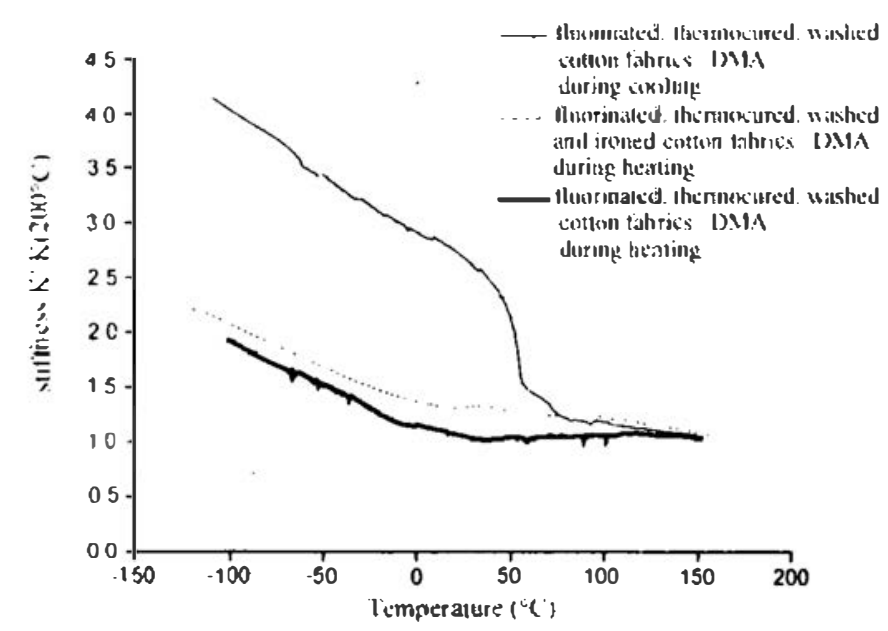

Figtire 5. Evolution of stiffness as a function of temperaltupe för two tiuorinated treated coltons. One is thermocured and washed: LFMA has been performed on this sample during heating under vacuum at $I^{\circ} \mathrm{Cl}$ min up to $150^{\circ} \mathrm{C}$ before couling under vacuum at the same rate. The other is thermoxurted. washed and ironed: LFMA has been pertormed on this sample during healing under air at $1^{\circ} \mathrm{C} / \mathrm{min}$ up $10150^{\circ} \mathrm{C}$. From these results. the quantitative infuence on stiffiness of. on one hand. the furmation of bridges between tibers and. on the other hand, an improved polymer/cotion adhesion can be appreciated. 
during cooling at the same rate. In Figure 5 , it appears that a strong increase in stiffness of more than $100 \%$ is effectively noticed after annealing at $150^{\circ} \mathrm{C}$ when any manipulation is carrefully avoided. The main contribution to increased stiffness during ironing is then the formation of resin bridges between the tibers rather than the improved adhesion between the cotton and the fluorinated resin. This result is in good agreement with the previous evaluation of individual fiber stiffness increases after coating: much smaller than that measured experimentally on textiles, the coating related stiffness increase must be attributed almost entirely to the formation of resin bridges between the cotton fibers.
Confirming De-and Re-wetting Mechanisms bv MEB. $A F M$, and XPS

We further studied the differences so evident between the coated textiles before and after ironing with MEB and AFM experiments. After three washings at $40^{\circ} \mathrm{C}$. some minor fibrillation of cotton fibers was evident from MEB. Compared with a coated textile after curing (Figure (6d) there was also a strong increase in roughness (Figure be) observed by AFM at a smaller scale. This increase is of the order of magnitude of the polymer coating $(\sim 500 \mathrm{~nm})$ and can be attributed to a massive de-wetting of the fluorinated resin during washing. According to Figure $6 f$.

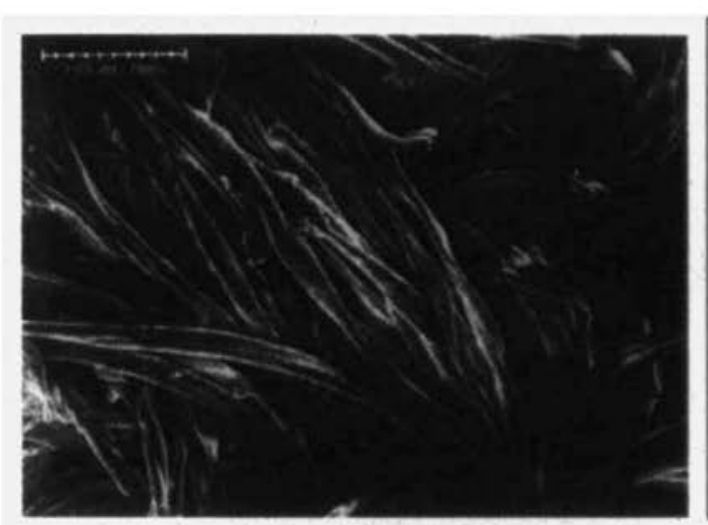

(a)

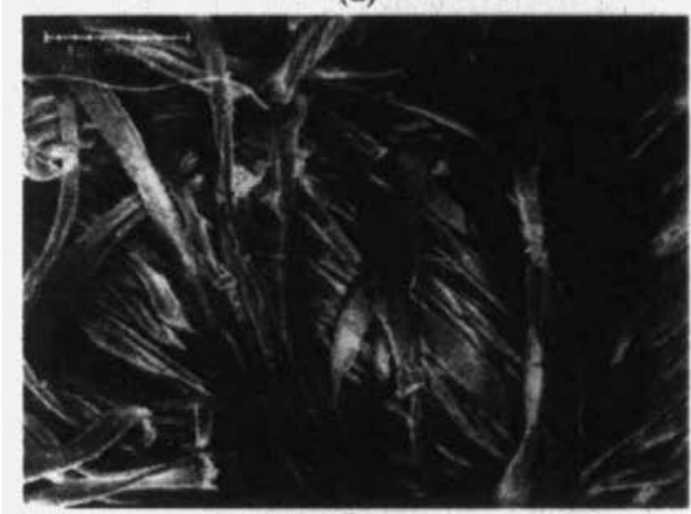

(b)

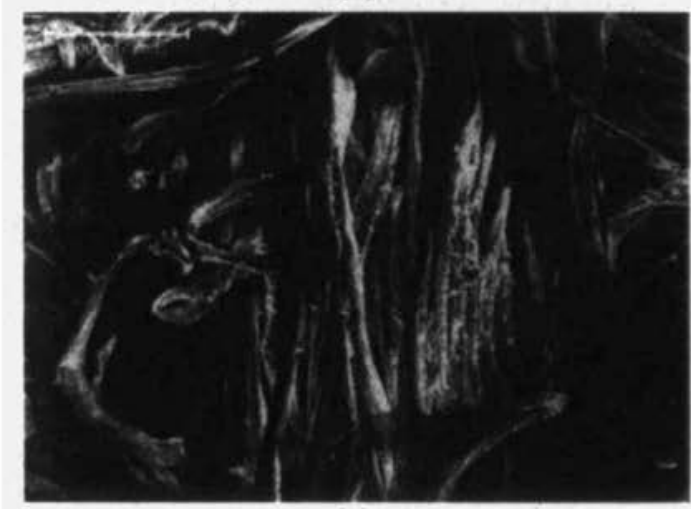

(c)

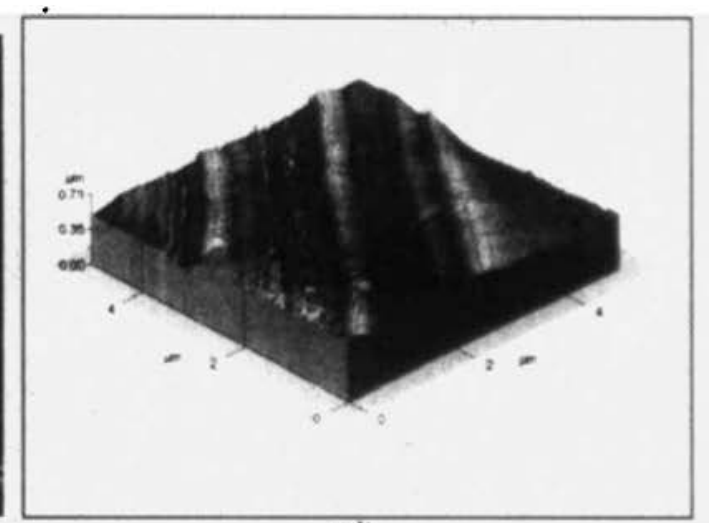

(d)

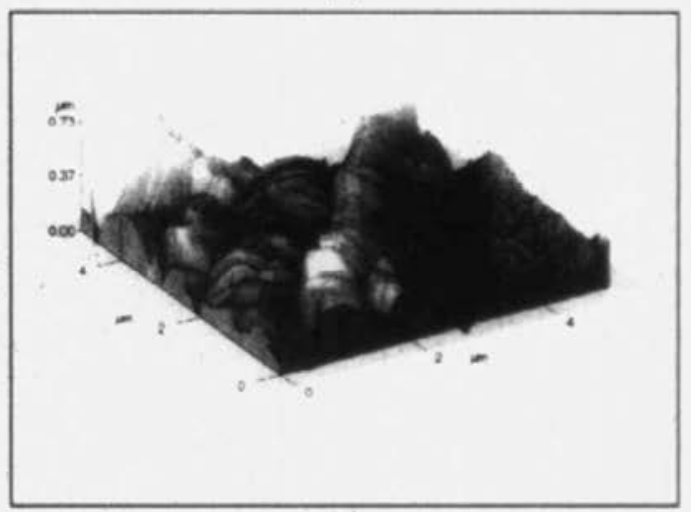

(e)

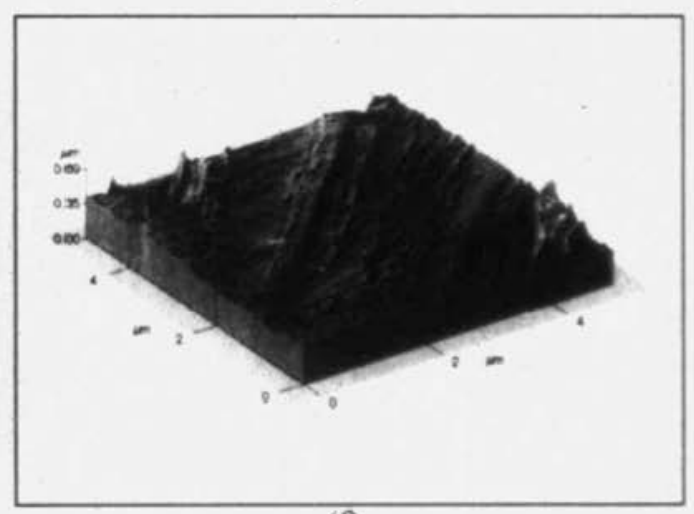

(f)

Figure 6. MEB (left) and AFM (right) investigations. Evolution of the morphology of the pulymer-coaced fibers during washing and ironing: (a and d) colated textile after cuing. (b and e) polymer coated textive afier washing. ( $c$ and $t$ ) polymer coated textile after washing and ironing. 
TABLE II. Surface $O$ and F atomic contents obtained by XPS analysis of cured/washed/ironed fiuorinated resin-treated cotton fabrics obtained with latex A padded with a polymer content of $150 \mathrm{~g} / \mathrm{l}$. Comparison with volume atomic contents obtained by chemical method.

\begin{tabular}{|c|c|c|c|}
\hline & $\begin{array}{l}\text { Volume fluorine comtent } \\
\text { (measured by chemical metbod) }\end{array}$ & $\begin{array}{l}\text { Surface uxygen content } \\
\text { (measured by XPS) }\end{array}$ & $\begin{array}{l}\text { Surface fluorine content } \\
\text { (measured by XPS) }\end{array}$ \\
\hline After curing & $8600 \pm 800 \mathrm{ppm}$ & $x_{0}^{\text {iunng }}=(5.5 \pm 0.05) \%$ al. & $x_{F}$ :urns: $=(54 \pm 5) \not$ al. \\
\hline After washing & $7.500 \pm 80(1) \mathrm{ppm}$ & $x_{0}{ }^{\text {wavhing }}=(11 \pm 0.1)$ \%ac & $x_{\mathrm{F}}^{\text {Haluting }}=(48 \pm 5) \%$ al. \\
\hline After ironing & $7900 \pm 800 \mathrm{ppm}$ & 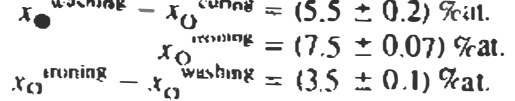 & 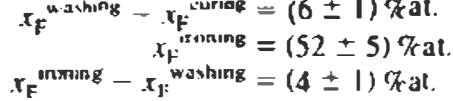 \\
\hline
\end{tabular}

ironing allows this de-wetting to be almost completely removed (fiber roughness of about $50 \mathrm{~nm}$ is equivalent to that observed before washing), whereas there is no effect on fibrillation during ironing (Figure $6 c$ ). Such observations are in perfect accord with the conclusions drawn from LPMS experiments.

The de-wetting suggested by mechanical spectroscopy is also confirmed by XPS experiments. In the case of coated and cured fabrics. the contents of $\mathrm{O}$ and $\mathrm{F}$ mea. sured by such a surface technique are, respectively, 5.5 $\pm 0.3 \%$ and $54 \pm 3 \%$ atomic, whereas the volume fluorine content measured by a chemical technique is about $8600 \pm 800 \mathrm{ppm}$ (Table II). In the hypothesis where the fibers are uniformly coated by the resin, XPS analysis is almost fully consistent with $\mathrm{O}$ and $\mathrm{F}$ polymer atomic content $(5.5$ and $44 \%$ ) of extemal fluorinated chains. That means that the analysis depth of XPS is hardly less than the order of magnitude of a lateral chain $\left(-\mathrm{CO}-\mathrm{O}-\left(\mathrm{CH}_{2}\right)_{2}-\mathrm{C}_{8} \mathrm{~F}_{17}\right)$. Now. a comparison of the surface composition of differently treated textiles (Table II) shows a decrease in fluorine content and an increase in oxygen during washing. In accord with previous results in the literature. these modifications ane almost suppressed after ironing. However, variations in the surface chemical composition obtained here are far less important [25] than those found in the literature. This is explained by less washing efficiency, consisting here of

TABLE ItI. Perfurmance of fluorinated resin-coated cotton after heat treatment and washing obtained from tatex $A\left(T_{m}=50^{\circ} \mathrm{C}\right)$ and latex $\mathrm{B}\left(T_{m}=80^{\circ} \mathrm{C}\right)$.

\begin{tabular}{|c|c|c|c|c|}
\hline & \multicolumn{2}{|c|}{$\begin{array}{l}\text { Water resistance. } \\
\text { AATCC } 22 \text { Tesi } \\
\text { (spray test) }\end{array}$} & \multicolumn{2}{|c|}{$\begin{array}{l}\text { Oil resistance. } \\
\text { AATCC } 118 \text { Tesi }\end{array}$} \\
\hline & $60 \mathrm{~g} / 1$ & $150 \mathrm{~g} / 1$ & $60 \mathrm{~g} / 1$ & $150 \mathrm{~g} / 1$ \\
\hline \multicolumn{5}{|l|}{ Latex $A$} \\
\hline After annealing & 100 & 100 & 6 & 6 \\
\hline After washing & 50 & 70 & 5 & 5 \\
\hline After ironing & 70 & 90 & 6 & 6 \\
\hline \multicolumn{5}{|l|}{ Latex B } \\
\hline After annealing & 100 & - & 6 & - \\
\hline After washing & 90 & - & 6 & - \\
\hline After ironing & 100 & - & 6 & - \\
\hline
\end{tabular}

only three cycles instead of thirty [25]. The evolution of surface fluorine content fluctuations after washing is generally attributed to the rotation of fluoroalkyl groups into the polymer substrate to decrease repulsive $-\mathrm{C}_{\mathrm{x}} \mathrm{F}_{17}$ ' water interactions.

From the combination of all these results. we propose a more relevant interpretation for such chemical evolution. Schematically illustrated in Figure 7 , it is based on the massive de-wetting occurring during washing. Since XPS provides surface information. the fluorinated polymer contribution to the XPS spectrum obviously decreases during washing, whereas that of cellulose increases in opposite proportion. The cellulose does not contain fluorine but oxygen in large amounts. Thus, during washing the measured surface $F$ content is expected to decrease, whereas that of $\mathrm{O}$ is expected to increase, corresponding effectively to the experimental results. Similarly, F and $O$ contents measured by XPS respectively increase and decrease during ironing. In fact, $\mathrm{F}$ and $\mathrm{O}$ content variations can be completely explained in the framework of a simple quantitative surface analysis model. If one denotes $x$ as the ratio.

$$
x=\frac{N_{-}^{\text {cellulore }}}{\left(N^{\text {eellulise }}+N^{\text {resin }}\right)} \text {, }
$$

where $N^{\text {relluluse }}\left(N^{\text {resin }}\right)$ is the number of cellulose (resin) atoms analyzed by XPS. Such a ratio is related to the proportion of the surface on which the cellulose is emerging: it would be equal only if the analysis depth and the atomic density in cellulose and resin were the same. The ratio $x$ can be obtained from the analyzed $O$ or $F$ contents, $x_{0}$ and $x_{1}$, according to the following equations:

$$
x_{0}=x x_{0}^{\text {cellulose }}+(1-x) x_{0}^{\text {resin }}
$$

or

$$
x_{\mathrm{F}}=x x_{\mathrm{F}}^{\text {cellulore }}+(1-x) x_{\mathrm{F}}^{\text {resin }} .
$$

In these equations, the $\mathrm{O}$ and $\mathrm{F}$ contents in cellulose and in resin, $x_{0}^{\text {cellulore }}=0.50, x_{0}{ }^{\text {resin }}=0.055 . x_{F}^{\text {cellulose }}=0$. $x_{F}^{\text {resin }}=0.54$, are determined from specific XPS experi- 


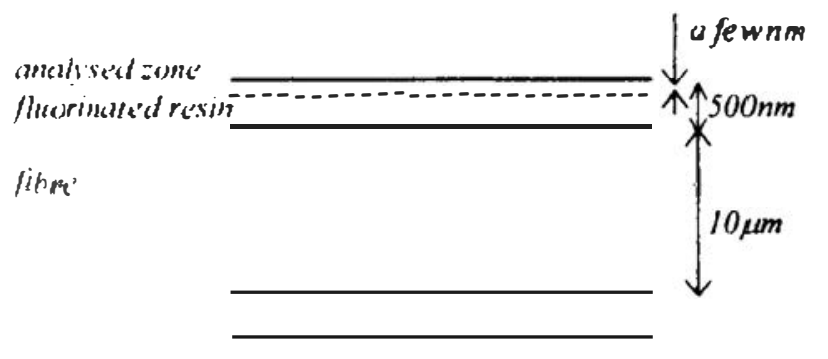

humegencenss souting of fibres after annealing
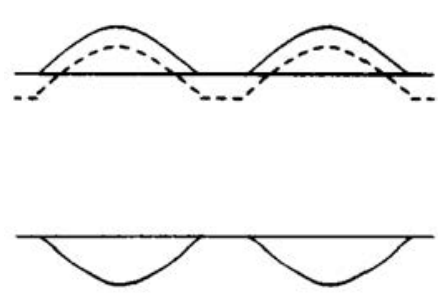

dewelting of Pworinared resin afoer washing

FIGURE 7. Schematic representation of the analyzed surface with XPS in the case of a uniform polymer corating (left) and in the case of massive polymer de-wetting on the fibens surface (right).

ments performed on pure cellulose and pure resin. From the XPS results of Table II, the $x$ decrease during washing is equal to $12 \pm 1 \%$ from 0 surface content variations and to $11 \pm 3 \%$ from F surface ones. Similarly, the $x$ increase during ironing is equal to $8 \pm 1$ or $8 \pm 3 \%$ by considering either $\mathrm{O}$ or $\mathrm{F}$ surf ace content variations. Note that. on the other hand, the rotation of the fluoroalkyl groups should not account for the observed variations of the $\mathrm{O}$ atomic content during laundering, since pure rotations would not involve any $O$ content change. The model based on de- and re-wetting is then fully consistent with all the experimental XPS results, so the wetting mechanisms are certainly more relevant than those relative to the rotation of fluoroalkyl groups to explain the modification of coated textiles during washing and ironing. In rather good agreement with AFM observations. the proportion of cellulose surface submitted to de-coating during washing is then around $11-12 \%$, and that undergoing re-coating during ironing is about $8 \%$.

\section{Improving Fabric Behavior DLring Laundering}

From our interpretation of mechanisms involved dur. ing washing and ironing, the behavior of fabrics during laundering could be improved by adapting some fluorinated resin characteristics. The more obvious interpretation is probably the increase in the polymer melting temperature above the washing temperature. The impact of such a solution is shown in Figure 8, which exhibits the temperature evolution of the complex modulus of two fluorinated resin-coated cottons with melting temperatures of, respectively, $50^{\circ} \mathrm{C}$ (latex A) and $80^{\circ} \mathrm{C}$ (latex $B$ ): indeed. the higher remaining stiffness after washing of fabrics coated with latex $B$ should indicate a better resistance to de-wetting. This indication is reinforced by an AFM comparison of cotton fabrics obtained from latex A (Figure 6) and latex B (Figure 8), showing that the appearance of fabric roughness during washing and iron- ing can be strongly decreased by coating with latex $B$ rather than with latex $A$.

Finally, we evaluated the water and oil resistance of the fabrics after curing. washing. and ironing according to the AATCC 22 (water repellency) and AATCC- 118 (oil repellency) tests for the two latices. All the values obtained in Table III are consistent with previous results and justify the use of the chosen combination of tech. niques for discrimination of the phenomena involved during the successive fabrication and laundering. First. there is a decrease in the water and oil repellency rating during washing. The higher the fluorinated polymer content ( $150 \mathrm{~g} / \mathrm{l}$ instead of $60 \mathrm{~g} / \mathrm{l})$. the higher the water repellency rating ( 70 instead of 50 ), which is explained by the larger remaining polymer-coated area of fibers af ter formation of polymer droplets during washing. The decreased water resistance rating during washing is also smaller for coating with latex B. which confirms a more difficult de-wetting of latex in the case of a high melting temperature. For latex B. the oil. resistance rating is not even modified during washing. Finally. there is some recovery of both water and oil repellency properties during ironing. which is in perfect agreement with all our experimental techniques and intespretations.

\section{Conclusions}

We have investigated the effects of heat treatments and washing on the mechanical and chemical evolution of cotton fabrics coated with fluorinated resin by means of a combination of mechanical (LFMA). surface chemical (XPS), and microstructural (AFM) lechniques. We propose a new explanation for the limitation of textile durability to laundering: during washing. the fluoroalkyl groups rotation into the polymer substrate is not as imporant as claimed in the literature. We show. on the contrary, that it is the massive de-wetting of the polymier coating. suggested by XPS, evidenced by LFMA, and directly ob- 


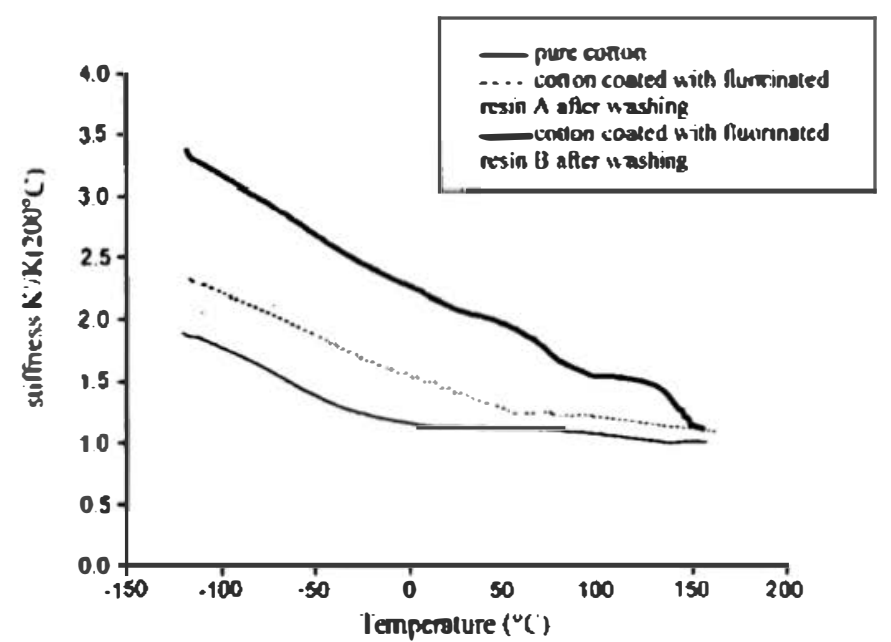

(a)

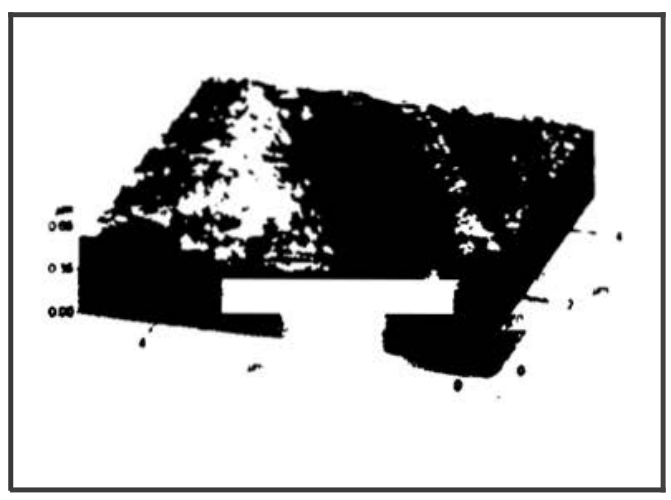

(b)

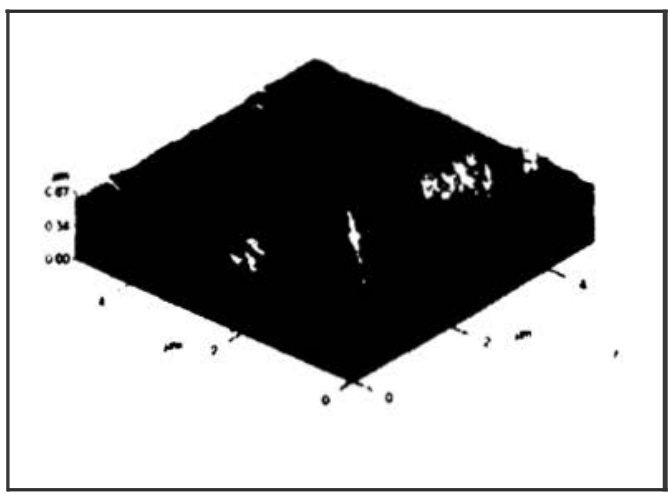

(c)

Figure 8. Stiffness (a) of two washed. fluosinated, resin-coated cotton fabrics (with melting temperatures of 50 and $80^{\circ} \mathrm{C}$. respectively) as a function of temperature during heating up (1) $1(x)^{\circ} \mathrm{C}$ at $1^{\circ} \mathrm{C} / \mathrm{min}$ under air. AFM investigations on a cotton padded with latex B. thernally cured and washed (b) then iruned (c).

served with AFM. which constitutes the major contribution to the diminished repellency of washed fabrics.

In this paper, the complementary interests of low frequency torsion mechanical spectrometry for studies of fuorinated coated cotton are evident. In particular, LFMA enables the relative contributions of the mechanisms involved in textile laundering evolution to be discriminated: they are the fonnation of polymer bridges between the fibers and the cotton/polymer adhesion strength. It also appears from LPMA that a satisfying compromise betwoen water- and oil-repellency and fabric drapability can be easily found. Finally, we conclude that textile durability may be increased in the most satisfying way by controlling the relative values of washing and resin melting temperatures.

\section{Literature Cited}

I. Arunyadej. S., Mitchell, R., Walton, J., and Carr. C. M.. An Investigation into the Effect of Laundering on the
Repellency Behaviour of a Fluorochemical-treated Cotton Fabric. J. Texsile Inst. 89, 696-702 (1998).

2. Ashby. M. F., "Materials Selection in Mechanical Design." Pergamon Press. U.K.. 1993, p. 28.

3. Blackwell. J.. Kolpak. F. J.. and Gardner. K. H.. Structure of Native and Regenerated Celluloses, in "Cellulose Chemistry and Technology," American Chemical Suciety, Washington. DC, 1977. pp. 42-55.

4. Brandrup. J.. and Immergut. E. H., "Polymer Handbook." 3rd ed. 1989. pp. VII7-VI59.

5. Briggs. D.. and Seah. M. P.. "Practical Surface Analysis by Auger and X-ray Photoelectron Spectroscopy. John Wiley \& Sons. NY. 1985. pp. 181-216.

6. Corpart. J. M.. and Dessaint. A.. Textilappreturen auf Fluorbasis. Melliand Texrilber. 78, El35 (1997).

7. De Crevoisier. G., Fabre. P.. Corpart. J. M., and Leibler. L.. Switchable Tackiness and Wettability of a Liquid Crystalline Polymer. Science. 285, 1246-1249 (1999).

8. Etienne. S.. Cavaillé. J. Y.. Perez, J.. Point. R., and Salvia. M. Automatic System for Micromechanical Properties. Rev. Sci. Instrum. 53-8, 1261-1266 (1982). 
9. Etienne. S. Cavajllé. J. Y.. Perez., J., and Salvia. M., Automatic System for Micromechanical Properties Analysis. J. Phys. 42, C5-1129-C5.1134 (1981).

10. Juhué. D.. and Lang. J.. Latex Film Formation in the Presence of Organic Solvents. Macromolecules 27, 695701 (1994).

11. Kauzmann. W.. Factors in Interpretation of Protein Denaturation. Adv. Prosein Chem. 14, 37-47 (1959).

12. Kondo. T. Hydrogen Bonds in Regioselectively Substicuted Cellulose Derivatives. J. Polym. Sici. B Polym. Phis. 32. 1229-1235 (1994).

13. Lakes, R. S.. "Viscuelastic Solĩd.” CRC Press. Boca Raton, FL. 1999, 77-86.

14. Linemann. R.. Gorenberg. A.. Bar. G.. Cantow. H. J., and Mülhaupt. R.. Synthesis of Fluorine-containing Dispersions and an Environmental Scanning Electron Micruscope Analysis of Their Morphology When Applied to Cocton Fabrics. J. Coasings Technol. 69, 77-81 (1997).

15. Montes, H. Cavaillé. J. Y.. and Mazeau. K.. Secondary Mechanical Relaxations in Amorphous Cellulose. Macromolecules 30, 6977-6984 (1997).

16. Moulder. J. F.. Stickle. W. F.. Sobol. P. E.. and Bombern. K. D.. "Handbook of X-ray Photvelectron Speciroscopy" (a ref erence book of standard spectra for identilication and interpretation of XPS data). Perkin-Elmer Corporation Physical Electronics Division. pp. 40-41 for information about C. pp. 44-45 for O. and pp. 46-47 for F.

17. Platé. N. A.. Sibaiev. V. P., Petrukhin. B. S.. and Kargin. V. A.. Structure of Comb-shaped Polymers with Fluorocarbon Side Groups, J. Polym. Sci. Part C 23, 37 (1968).

18. Postnikov, Belko V. N.. Darinskii. B. M.. and Sharshakov. 1. M.. Appar dtus for Fatigue Testing and Measuring Internal Friction in Metals. Fi:- Metallor Meralloved 27, 141 (1969).

19. Rowland. S. P.. Solid-liquid Interactions: Inter- and Intracrystalline Reactions in Cellulose Fibers, from the symposium "Water in Polymers". cosponsored by the ACS Macromolecular Secretariat and the Division of Biological Chemistry at the 178th meeting of the American Chemical Society. 1980. pp. 205-233.
20. Scofield, J. H.. Harree-Slater Subshell Photoionizacion Cross-sections at 1254 and $1487 \mathrm{eV}$. J. Electron Spectrosc. Relared Phensm. 8, 129-137 (1976).

21. Seah. M. P.. and Dench. W. A.. Quantitative Elocton Spectroseppy of Surfaces: A Sundand Data Base for Elocton Inelastic Mean Free Path. Surt: Interface Anal. 1, 2 (1979).

22. Seah. M. P.. The Quantitative Analysis of Surfaces by XPS: A Review. Surf. Interf ace Anal. 2 222 (1980). The transmitted peak area intensity/kinetic energy relationship depends on the spectrometer used. With SSI-SX 100. the transmission function depends on $E_{\text {. }}{ }^{\text {. }}$. while with ESCALAB $200 R$. it depends on $E_{\text {, } \pi / .}$ " .

23. Shenouda. S. G.. "Applied Fiber Science. Volume 3," Academic Press. NY. 1979. PP. 275-298.

24. Shirley. D. A.. High Resolution X-ray Photoemission Spectrum of the Valence Bands of Cold. Phys. Rev: B V. 4709 (1972).

25. Tahara. M.. Nakashima. Y., Miyazaki, K.. and Takag̨ashi. T. Washing Durability of Cocton and Wool Fabrics Treated by Plasma Polymerization of Perfluorocarbons. $J$. Texrile Machin. Soc. Jpn. 49, T166-TI74 (1996).

26. Tan. P.. Tong. L.. and Steven. G. P.. Modelling for Pre. dicting the Mechanical Properties of Textile Composites. Cimponsiles Pan A 28A, 903-922 (1997).

27. Wakida. T., Li. H., Sato, Y.. Kawamura. H., Ueda. M., Mizushima. H.. and Takekoshi. S.. The Effect of Washing and Heat Treatment on whe Surface Characteristics of Fluorocarbon Resin-treated Polyester. J. Soc. Dyers Colour.. 109 (1993).

28. Wakida. T.. Tokino. S. Niu, S., Ueda. M.. Mizushima. H.. and Takekoshi. S.. Effect of Crosslinking Agents on Water Repellency of Conon Fabrics Treaced wist Fluorocation Resin. Texsile Res. J. 64. 316-320 (1994).

29. Woirgard. J.. Sarrazin. Y.. and Chaumet. H. Apparatus for the Measurement of Internat Friction as a Function of Frequency Between $10^{\text {s }}$ and $10 \mathrm{~Hz}$. Rev. Sci. Insinen 481 10). 1322-1 325 (1977). 